\title{
BROWNIAN MOTION IN A WEDGE WITH VARIABLE SKEW REFLECTION
}

\author{
L. C. G. ROGERS
}

\begin{abstract}
Does planar Brownian motion confined to a wedge by skew reflection on the sides approach the vertex of the wedge? This question has been answered by Varadhan and Williams in the case where the direction of reflection is constant on each of the sides, but here we address the question when the direction reflected is allowed to vary. A necessary condition, and a sufficient condition, are obtained for the vertex to be reached. The conditions are of a geometric nature, and the gap between them is quite small.
\end{abstract}

\section{INTRODUCTION}

There has been a lot of recent interest in the behaviour of Brownian motion in some domain $D \subset \mathrm{C}$ with skew-reflection on the boundary-see [DeB, HLS, LeG, TW, VW, W85a, W85b] and, for multi-dimensional generalizations, [LS, SV, W87, ReW].

In [VW], the domain $D$ is taken to be a wedge $D=\left\{r e^{i \theta} ; 0 \leq \theta \leq \alpha, r>0\right\}$ and the direction of reflection is assumed to be constant on each of the edges of the wedge. A number of very natural questions arise about this process, one of which is "Will the process approach 0 ?" This question, and the much more difficult one, 'Is it possible to extend the process beyond its first hit on 0?" are completely answered in [VW] (see also [R] for a proof by excursion-theoretic methods).

The aim of this paper is to examine the question, "Will the process approach 0 ?" when the direction of reflection on the edges of $D$ are not assumed constant. This turns out to make the question considerably more difficult, so we shall confine ourselves here only to this problem and defer treatment of other related interesting questions until a subsequent paper.

To begin with, it is helpful to transform the problem via probabilistic and complex-analytical methods. Clearly, whether or not Brownian motion in $D$ can approach 0 is decided by the directions of reflection in a neighbourhood of 0 , so we lose no generality in assuming the direction of reflection is normal outside $\{z:|z|<1\}$. As is well known, the sample paths of Brownian

Received by the editors May 19, 1989.

1980 Mathematics Subject Classification (1985 Revision). Primary 30E25, 60J65, $60 \mathrm{~J} 50$.

Key words and phrases. Brownian motion with skew reflection, conformal transformation, recurrence, transience. 
motion map under an analytic function to the sample paths of a time-changed Brownian motion, and this remains true if the Brownian motion is reflected off the boundary of some domain. Thus, by applying the analytic function $z \rightarrow-1 / z^{\pi / \alpha}$, the region $D$ gets mapped into the upper half-plane $\mathbf{H}$, the point 0 gets mapped to $\infty$, and the point $\infty$ gets mapped to 0 . We shall henceforth take the problem in this form: one considers a Brownian motion in $\mathbf{H}$ reflected off $\mathbf{R} \equiv \partial \mathbf{H}$ in a variable direction $\theta(x), x \in \mathbf{R}$, and asks whether the process is transient. The angle $\theta(x)$ is measured in a clockwise direction from the inward-pointing normal, so that $-\pi / 2 \leq \theta(x) \leq \pi / 2$ for all $x$.

We make the following assumptions about the function $\theta$ :

(1.i) $\theta$ is $C^{1}$ with bounded derivative;

(1.ii) for some $A,|\tan \theta(x)| \leq A(1+|x|)$ for all $x$.

These conditions can probably be relaxed slightly, but let us make a few remarks on them now. The obvious way to construct a Brownian motion in $\mathbf{H}$ with the specified skew reflection is to take independent real Brownian motions $B, B^{\prime}$, to fix the starting point $x_{0}+i y_{0} \in \mathbf{H}$, and define

$$
\begin{gathered}
L_{t} \equiv \sup _{s \leq t}\left(-y_{0}-B_{s}^{\prime}\right)^{+}, \quad Y_{t} \equiv y_{0}+B_{t}^{\prime}+L_{t}, \\
X_{t}=x_{0}+B_{t}+\int_{0}^{t} \tan \theta\left(X_{s}\right) d L_{s} .
\end{gathered}
$$

The stochastic differential equation (2.ii) for $X$ will have a solution provided (see, for example, [RW, §V.11]) the conditions (1.i) and (1.ii) hold. Thus something similar to (1) is needed to ensure the existence of such a skew-reflecting Brownian motion. Now observe that if $f: \bar{H} \rightarrow \mathbf{R}$ is $C^{2}$, then, by Itô's formula,

$$
\begin{aligned}
d f\left(X_{t}, Y_{t}\right)= & \frac{\partial f}{\partial x}\left(X_{t}, Y_{t}\right) d X_{t}+\frac{\partial f}{\partial y}\left(X_{t}, Y_{t}\right) d Y_{t}+\frac{1}{2} \Delta f\left(X_{t}, Y_{t}\right) d t \\
= & \left\{\frac{\partial f}{\partial x}\left(X_{t}, Y_{t}\right) d B_{t}+\frac{\partial f}{\partial y}\left(X_{t}, Y_{t}\right) d B_{t}^{\prime}\right\}+\frac{1}{2} \Delta f\left(X_{t}, Y_{t}\right) d t \\
& +\left[\tan \theta\left(X_{t}\right) \frac{\partial f}{\partial x}\left(X_{t}, Y_{t}\right)+\frac{\partial f}{\partial y}\left(X_{t}, Y_{t}\right)\right] d L_{t} .
\end{aligned}
$$

The term in the curly brackets is a continuous local martingale, so $f\left(X_{t}, Y_{t}\right)$ is a local martingale if and only if $\Delta f=0$, and $f$ satisfies the boundary condition

$$
\tan \theta(x) \frac{\partial f}{\partial x}(x, 0)+\frac{\partial f}{\partial y}(x, 0)=0, \quad \forall x \in \mathbf{R} .
$$

If now $f$ were the imaginary part of analytic $F=g+i f$, this boundary condition says that, on $\mathbf{R}$, the real part of $e^{-i \theta} F^{\prime}$ is zero. This suggests that we consider an analytic function whose derivative has argument $\theta$ on $\mathbf{R}$. Thus we define the analytic function $\psi: \mathbf{H} \rightarrow \mathbf{C}$ by

$$
\psi(z) \equiv \exp \left[\int_{-\infty}^{\infty} \frac{\theta(x) d x}{\pi}\left\{\frac{1}{x-z}-\frac{x}{1+x^{2}}\right\}\right]
$$


The assumptions (1) guarantee that $\psi$ extends continuously to $\overline{\mathbf{H}}=\mathbf{H} \cup \mathbf{R}$, $\operatorname{Re} \psi(z)>0$ for all $z \in \overline{\mathbf{H}}$, and for $x \in \mathbf{R}, \arg \psi(x)=\theta(x)$. Define next the analytic function

$$
\Psi(z)=\int_{0}^{z} \psi(\omega) d \omega, \quad z \in \overline{\mathbf{H}} .
$$

This technique has proven useful in computations when $\theta$ is assumed to have some tractable form, such as piecewise constant; see, for example, [N, or TW].

We investigate properties of $\Psi$ in $\S 2$; for example it is shown there that $\Psi$ is $1-1$, and if $a+i b \in \Psi(\mathbf{H})$ then $a+i b^{\prime} \in \Psi(\mathbf{H})$ for all $b^{\prime}>b$. The function $\Psi$, and, more especially, the shape of the domain $\Psi(\mathbf{H})$ are the key to understanding the problem. This is because $\Psi$ twists around the directions of reflection on the boundary so that the reflection on the boundary of $\Psi(\mathbf{H})$ is always in the (upward) imaginary direction. Thus the problem can be seen as one of deciding the transience of Brownian motion in $\Psi(\mathbf{H})$ with upward reflection on the boundary. We show in $\S 3$ the main result that

(5.i) the process is recurrent if $\Psi(\mathbf{H})$ contains a wedge of angle $\geq \pi$;

(5.ii) the process is transient if $\psi(\mathbf{H})$ is contained in a wedge of angle $<\pi$.

In terms of the function $\theta$, if we write $i+\Psi(t) \equiv \rho(t) \exp \{i \beta(t)\}, t \in \mathbf{R}$, the polar representation, where $-\pi / 2<\beta(t)<3 \pi / 2$ for all $t$, then

(6.i) $\lim \sup _{t \rightarrow \infty} \beta(t)+\pi<\liminf _{t \rightarrow-\infty} \beta(t) \Rightarrow$ the process is recurrent;

(6.ii) $\liminf _{t \rightarrow \infty} \beta(t)>\lim \sup _{t \rightarrow-\infty} \beta(t)-\pi \Rightarrow$ the process is transient.

The dependence of these criteria on $\theta$, via (3) and (4), is somewhat unwieldly, but the cases where $\theta$ has limiting directions are easily handled by this. In particular, it is easy to deduce from (5.i)-(5.ii) the following result.

Corollary. (i) The process is recurrent if $\limsup _{t \rightarrow \infty} \theta(t)<\liminf _{t \rightarrow-\infty} \theta(t)$.

(ii) The process is transient if $\liminf _{t \rightarrow \infty} \theta(t)>\lim \sup _{t \rightarrow-\infty} \theta(t)$.

We should remark here that similar methods are used by Dynkin [D], and Malyutov $[\mathrm{M}]$ in their work on Brownian motion with skew reflection. The problem considered in these papers can be viewed as the problem of Brownian motion in the unit disc with a direction of reflection on the boundary given by a $C^{1, \alpha}$ vector field which is tangential only at a finite set of points. The vector field may sometimes point outward, but the boundary condition of a zero derivative in the direction of the vector field remains well-defined; all that happens (in our language) is that at a finite set of points $\theta$ can jump from $\pi / 2$ to $-\pi / 2$, or vice versa. The situation which we are dealing with differs from that of Dynkin and Malyutov in that we are allowing only one singularity in the vector field on the boundary, but that singularity may be far worse. 


\section{Properties of THE FUnCTION $\Psi$}

We establish here a number of basic properties of $\Psi$ and of $\Psi(\mathbf{H})$.

Lemma 1. The function $\Psi: \overline{\mathbf{H}} \rightarrow \Psi(\bar{H})$ is $1-1$.

Proof. Suppose $z_{1}, z_{2}$ are distinct points of $\overline{\mathbf{H}}$ such that $\Psi\left(z_{1}\right)=\Psi\left(z_{2}\right)$. If $\gamma$ is the curve $\gamma(t)=(1-t) z_{1}+t z_{2} \quad(0 \leq t \leq 1)$, then

$$
0=\Psi\left(z_{2}\right)-\Psi\left(z_{1}\right)=\int_{\gamma} \psi(\omega) d \omega=\left(z_{2}-z_{1}\right) \int_{0}^{1} \psi(\gamma(t)) d t .
$$

Since $\operatorname{Re} \psi>0$ throughout $\overline{\mathbf{H}}$, the real part of the integral is positive, so, in particular, the integral is a nonzero complex number, yielding a contradiction.

To understand more fully the shape of $\Psi(\mathbf{H})$, let us first make the simplifying assumption

$$
\inf \{\operatorname{Re} \Psi(t): t \in \mathbf{R}\}=-\infty, \quad \sup \{\operatorname{Re} \Psi(t): t \in \mathbf{R}\}=+\infty .
$$

The real part of the curve $\Psi: \mathbf{R} \rightarrow \mathbf{C}$ is always increasing, since $\operatorname{Re} \Psi^{\prime}(t) \equiv$ $\operatorname{Re} \psi(t)>0$ everywhere. Using properties of Brownian motion, we shall deduce that

$$
\Psi(\mathbf{H})=\{\Psi(t)+i v: t \in \mathbf{R}, v>0\} \equiv D_{+} .
$$

The argument runs as follows. Since $\Psi$ is one-one and an analytic function, $\Psi(\mathbf{H})$ is an open, arcwise-connected, simply-connected subset of $\mathbf{C}$. Now, since $\operatorname{Im}\{\Psi(x+i y)-\Psi(x)\}>0$, for any $x \in \mathbf{R}, y>0$ (recall that $\operatorname{Re} \psi>0$ ), and $\partial \Psi / \partial y$ is orthogonal to $\partial \Psi / \partial x$ on $\mathbf{R}$, it must be that $\Psi(\mathbf{H}) \subseteq D_{+}$, for, if not, there would be somie $x+i y \in \mathbf{H}$ such that $\Psi(x+i y) \notin D_{+}$; this would imply that the image under $\Psi$ of the curve $\{x+i t: 0 \leq t \leq y\}$ met the boundary $\Psi(\mathbf{R})$ at some point other than $\Psi(x)$, which would contradict the injectivity of $\Psi$.

Thus $\Psi(\mathbf{H}) \subseteq D_{+}$, and now we can argue that $\Psi(\mathbf{H})=D_{+}$. Suppose on the contrary that $D_{+} \mid \Psi(\mathbf{H})$ contained some point $\xi_{0}$. Because $\Psi(\mathbf{H})$ is simplyconnected, there is a curve $\Gamma$ from $\xi_{0}$ to $\propto$ not meeting $\Psi(\mathbf{H})$. Since $\Gamma$ is nonpolar for Brownian motion, if one started a Brownian motion at some point $\omega_{0} \in \Psi(\mathbf{H})$, there is a positive probability that the process will reach $\Gamma$ before it reaches $\Psi(\mathbf{R})$. Thus there is a positive probability that the process will exit $\Psi(\mathbf{H})$ at some point not in $\Psi(\mathbf{R})$ before ever reaching $\Psi(\mathbf{R})$. But recall that a skew-reflected Brownian motion started at $\Psi^{-1}\left(\omega_{0}\right)$ maps to (a time change of) skew-reflected Brownian motion in $\Psi(\mathbf{H})$. Thus there would be a positive probability that this Brownian motion in $\mathbf{H}$ would never reach $\mathbf{R}$, which is impossible. Hence we would have established (9) under assumption (8).

To study the other possibilities, we can, for simplicity and without loss of generality, assume $\inf \{\operatorname{Re} \Psi(t): t \in \mathbf{R}\}=-\infty$ and define $\sigma \equiv \sup \{\operatorname{Re} \Psi(z): z \in$ $\mathbf{H}\}, \eta \equiv \sup \{\operatorname{Re} \Psi(x): x \in \mathbf{R}\}$. Next we consider what can be said if $\sigma=\eta<$ $\infty$. In this case, it has to be that $\sup _{t>0} \operatorname{Im} \Psi(t)=+\infty$. We can prove this as before using Brownian motion, as follows. If $\sup _{t>0} \operatorname{Im} \Psi(t)<\infty$, it would be possible to start a Brownian motion at $x+i y \in \Psi(\mathbf{H})$, where $\eta-\varepsilon<x<\eta$ 
and $y$ is very large, and with positive probability this Brownian motion would reach $\{\operatorname{Re}(z)=\eta\}$ without hitting $\Psi(\mathbf{R})$. But Brownian motion $Z$ in $\mathbf{H}$ with skew-reflection $\theta$ keeps on hitting $\mathbf{R}$-there is no last hit on $\mathbf{R}$. Thus $\Psi(Z)$ keeps on hitting $\Psi(\mathbf{R})$, a contradiction. In summary, then, (9) also holds if $\sigma=\eta<\infty$.

The last case to be considered is that in which $\eta<\sigma$. Take some $\xi_{0} \in \Psi(\mathbf{H})$, with $\operatorname{Re}\left(\xi_{0}\right)>\eta$, and notice that there is a smooth curve in $\Psi(\mathbf{H})$ joining $\xi_{0}$ to 0 (because there is a smooth curve in $\mathbf{H}$ joining $\Psi^{-1}\left(\xi_{0}\right)$ to 0 ). This implies that $\sup \{\operatorname{Im} \Psi(x): x \geq 0\}$ must be finite. Next, it must be that $\Psi(\mathbf{H})$ contains $\{z: \operatorname{Re}(z)>\eta\}$, for, if not, there is some $\xi_{1} \notin \Psi(\mathbf{H})$ such that $\operatorname{Re}\left(\xi_{1}\right)>\eta$, and so, as before, there is a curve $\Gamma$ joining $\xi_{1}$ to $\infty$ not meeting $\Psi(\mathbf{H})$. But now we can argue as before: a Brownian motion in $\Psi(\mathbf{H})$ started at $\xi_{0}$ will with positive probability exit $\Psi(\mathbf{H})$ before reaching $\operatorname{Re}(\xi)=\eta$, which would mean that a Brownian motion in $\mathbf{H}$ could escape to infinity without ever reaching the real axis. This contradiction implies that $\Psi(\mathbf{H}) \supseteq\{z: \operatorname{Re}(z)>\eta\}$, and, in particular, $\sigma=+\infty$. A similar argument shows that one must also have $\inf \{\operatorname{Im} \Psi(x): x \geq 0\}=-\infty$. This gives a very clear picture of what $\Psi(\mathbf{H})$ looks like.

One final point to clear out of the way before proceeding is the assertion that $\Psi\left(X_{t}+i Y_{t}\right)$ is Brownian motion in $\Psi(\overline{\mathbf{H}})$ with vertical reflection on the boundary (here, $X+i Y$ is defined by $(2 . \mathrm{i})-(2 . \mathrm{ii}))$. The only small snag is that $\Psi$ is only $C^{1}$ on $\partial \mathbf{H}=\mathbf{R}$, so we apply Itô's formula to $\Psi\left(X_{t}+i Y_{t}+i \varepsilon\right)$ to see that, with $Z_{t} \equiv X_{t}+i Y_{t}$,

$$
\begin{aligned}
d\left(\Psi\left(Z_{t}+i \varepsilon\right)\right) & =\psi\left(Z_{t}+i \varepsilon\right) d Z_{t} \\
& =\psi\left(Z_{t}+i \varepsilon\right)\left(d B_{t}+i d B_{t}^{\prime}\right)+i \sec \theta\left(X_{t}\right) e^{-i \theta\left(X_{t}\right)} \psi\left(Z_{t}+i \varepsilon\right) d L_{t}
\end{aligned}
$$

so that, in the limit as $\varepsilon \downarrow 0$,

$$
d \Psi\left(Z_{t}\right)=\psi\left(Z_{t}\right)\left(d B_{t}+i d B_{t}^{\prime}\right)+i \sec \theta\left(X_{t}\right)\left|\psi\left(X_{t}\right)\right| d L_{t} .
$$

\section{CRITERIA FOR RECURRENCE OR TRANSIENCE}

As was explained in the Introduction, the recurrence or transience of the skew-reflecting Brownian motion in $\mathbf{H}$ is decided by what $\Psi(\mathbf{H})$ looks like.

Theorem 2. (i) If $\Psi(\mathbf{H})$ contains a wedge of angle $\geq \pi$, then the process is recurrent.

(ii) If $\Psi(\mathbf{H})$ is contained in a wedge of angle $<\pi$, then the process is transient.

Proof. (ii) We may without loss of generality assume that the wedge of angle $<\pi$ containing $\Psi(\mathbf{H})$ has vertex on the negative imaginary axis, since the wedge has to contain $i \mathbf{R}^{+}$. Let the vertex be at $-i b, b>0$, and suppose that $D_{0} \equiv \Psi(\mathbf{H})+i b$ is contained in the wedge

$$
\left\{z: \frac{\pi}{2}-\beta_{1} \leq \arg (z) \leq \frac{\pi}{2}+\beta_{2}\right\},
$$

where $\beta_{1}, \beta_{2}>0, \beta_{1}+\beta_{2}<\pi$. 


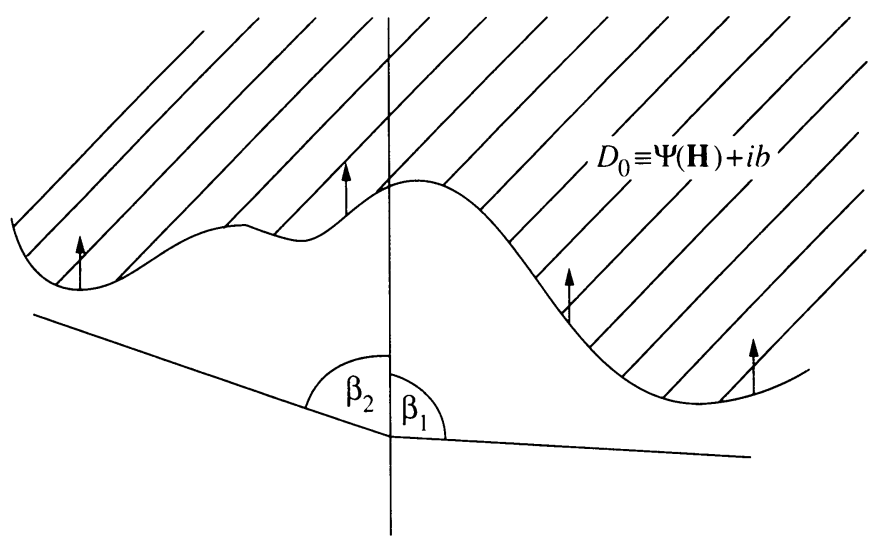

FIGURE 1

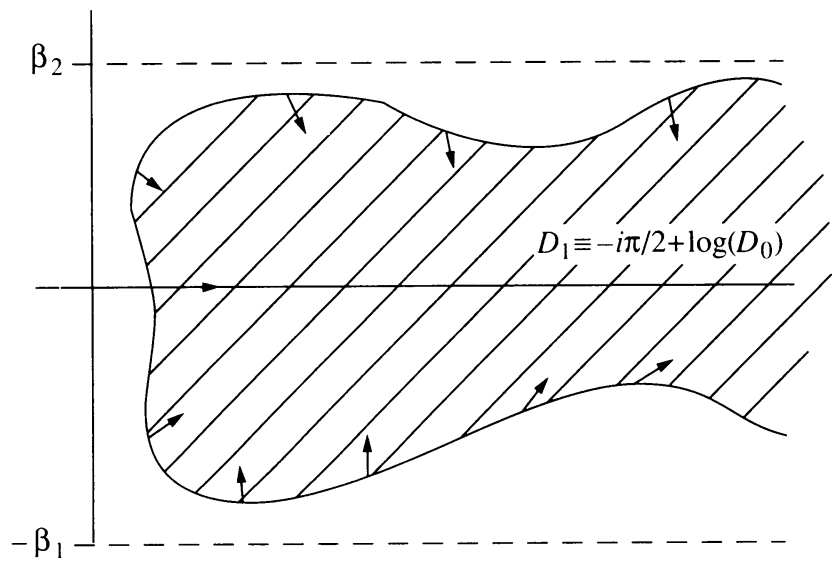

FIGURE 2

Let us define $g(z) \equiv-i \pi / 2+\log (z)$, where the cut of $\log$ is taken along the negative imaginary axis, so that $g$ maps $D_{0}$ to a region $D_{1} \subseteq\left\{z:-\beta_{1} \leq\right.$ $\left.\operatorname{Im}(z) \leq \beta_{2}\right\}$, and $g$ sends Brownian motion in $D_{0}$ with upward reflection on the boundary to a time-change of Brownian motion in $D_{1}$ with the corresponding reflection on $\partial D_{1}$. The direction of reflection at the point $\rho+i \theta \in \partial D_{1}$ is easily seen to be $\cos \theta-i \sin \theta$. The aim now is to prove that Brownian motion in $D_{1}$ with this reflection on the boundary is transient. This process solves the stochastic differential equation

$$
d \rho_{t}=d B_{t}+\cos \theta_{t} d L_{t}, \quad d \theta_{t}=d B_{t}^{\prime}-\sin \theta_{t} d L_{t},
$$

where $B, B^{\prime}$ are independent Brownian motions on $\mathbf{R}$, and $L$ is a continuous increasing process growing only when $\rho_{t}+i \theta_{t} \in \partial D_{1}$.

Now choose some $\gamma \in\left(\beta_{1}, \pi-\beta_{2}\right)$. Because $\theta_{t}$ lies always in $\left(-\beta_{1}, \beta_{2}\right)$, 


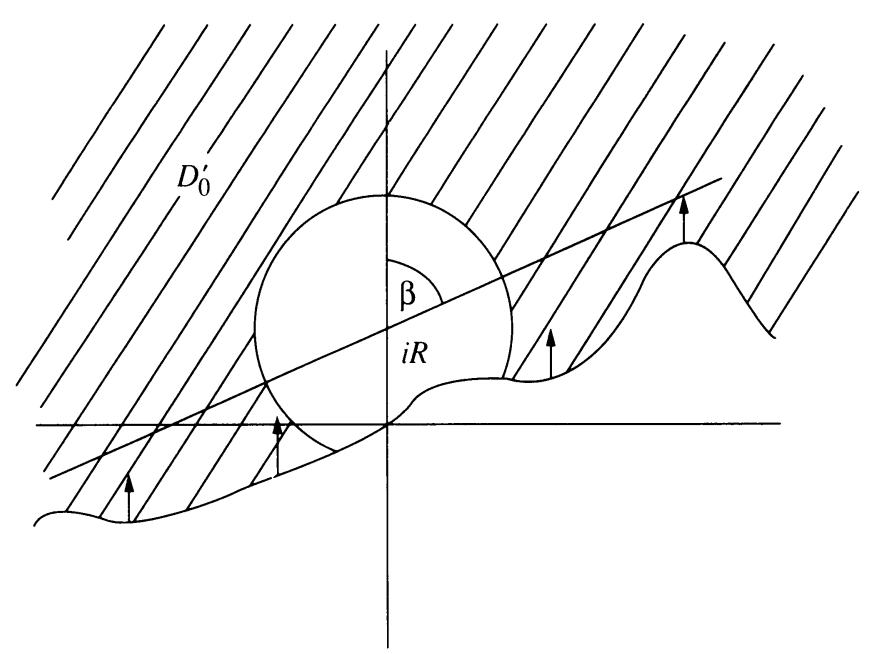

FIGURE 3

there is some $\varepsilon>0$ such that $\sin \left(\gamma+\theta_{t}\right) \geq \varepsilon$ always. Hence, from (10),

$$
\rho_{t} \sin \gamma-\theta_{t} \cos \gamma=B_{t} \sin \gamma-B_{t}^{\prime} \cos \gamma+\int_{0}^{t} \sin \left(\gamma+\theta_{s}\right) d L_{s},
$$

so, dividing by $t$ and letting $t \rightarrow \infty$, we deduce that

$$
\sin \gamma \liminf _{t \rightarrow \infty} t^{-1} \rho_{t} \geq \varepsilon \liminf _{t \rightarrow \infty} t^{-1} L_{t},
$$

since $\theta$ is bounded, and $B$ is $o(t)$. To estimate the right-hand side of (11), we use Itô's formula to give

$$
\theta_{t}^{2}-\theta_{0}^{2}=2 \int_{0}^{t} \theta_{s} d B_{s}^{\prime}-2 \int_{0}^{t} \theta_{s} \sin \theta_{s} d L_{s}+t
$$

so

$$
\lim _{t \rightarrow \infty} \frac{1}{t} \int_{0}^{t} 2 \theta_{s} \sin \theta_{s} d L_{s}=1 \text { a.s. }
$$

since the stochastic integral in (12) is $o(t), \theta$ being bounded.

Now observe that $\theta$ must lie in $(-\pi, \pi)$ so that, for some constant $C$, we have $0 \leq \theta_{s} \sin \theta_{s} \leq C$, and hence, from (13),

$$
\liminf _{t \rightarrow \infty} t^{-1} L_{t} \geq(2 C)^{-1} \text {. }
$$

Combined with (11), this implies that $\rho_{t} \rightarrow \infty$ a.s.

(i) It is enough to prove that if $\Psi(\mathbf{H})$ contains a wedge of angle $\pi$ (that is a half-space) then the process is recurrent. So let $i R$ be a point on the positive imaginary axis such that the half-space bounded below by the line through $i R$, making angle $\beta \in(0, \pi)$ with the upward imaginary axis in the clockwise sense, is contained in $\Psi(\mathbf{H})$. Consider now the domain $D_{0}^{\prime} \equiv \Psi(\mathbf{H}) \cap\{z:|z-i R|>$ $R+1\}$. If the cut of $\log$ is taken to be the negative imaginary axis, then 
$g(z) \equiv-i \pi / 2+\log (z-i R)$ maps $D_{0}^{\prime}$ to a subset $D_{1}^{\prime}$ of $\{z: \operatorname{Re} z>\log (R+1)$, $-\pi<\operatorname{Im} z<\pi\}$ and $D_{1}^{\prime}$ contains

$$
D_{2}^{\prime} \equiv\{z: \operatorname{Re} z>\log (R+1),-\beta<\operatorname{Im} z<\pi-\beta\} .
$$

Let $Z$ be Brownian motion in $D_{0}^{\prime}$ with upward reflection on $\Psi(\mathbf{R})$ absorbed when it reaches $\{z:|z-i R|=R+1\}$. The claim is that $Z$ will eventually reach this set. To see this, notice that, as before, after a time-change, $Z$ gets mapped by $g$ to a Brownian motion in $D_{1}^{\prime}$ with the specified direction of reflection, and absorption at the line $\operatorname{Re} z=\log (R+1)$. Thus the aim is to prove that Brownian motion with skew reflection in $D_{1}^{\prime}$ eventually gets absorbed at $\operatorname{Re} z=\log (R+1)$. However, as before, up until the time of absorption

$$
\begin{aligned}
& d \rho_{t}=d B_{t}+\cos \theta_{t} d L_{t}, \\
& d \theta_{t}=d B_{t}^{\prime}-\sin \theta_{t} d L_{t}
\end{aligned}
$$

(where $\rho+i \theta$ is the Brownian motion in $D_{1}^{\prime}$ ) and when $L$ grows, $\theta$ must be in either $(-\pi,-\beta]$ or $[\pi-\beta, \pi)$. Thus

$$
\rho_{t} \sin \beta-\theta_{t} \cos \beta=B_{t} \sin \beta-B_{t}^{\prime} \cos \beta+\int_{0}^{t} \sin \left(\beta+\theta_{s}\right) d L_{s},
$$

and $\sin \left(\beta+\theta_{s}\right) \leq 0$ whenever $L$ grows. If $\rho+i \theta$ did not eventually reach $\operatorname{Re} z=\log (R+1)$, then (15) would tend to $-\infty$, since $B_{t} \sin \beta-B_{t}^{\prime} \cos \beta$ is a Brownian motion, and $\int_{0}^{t} \sin \left(\beta+\theta_{s}\right) d L_{s}$ is decreasing. This would imply that $\rho$ was not bounded below, a contradiction.

Remarks. (i) The stochastic calculus analysis of $\rho+i \theta$ by which the theorem was proved may be replaced by an elegant comparison argument due to K. Burdzy and J.-F. Le Gall. To prove part (ii) of the theorem, the argument runs as follows. Let $C$ be a wedge of angle $<\pi$ containing $\Psi(\mathbf{H})$. The Brownian

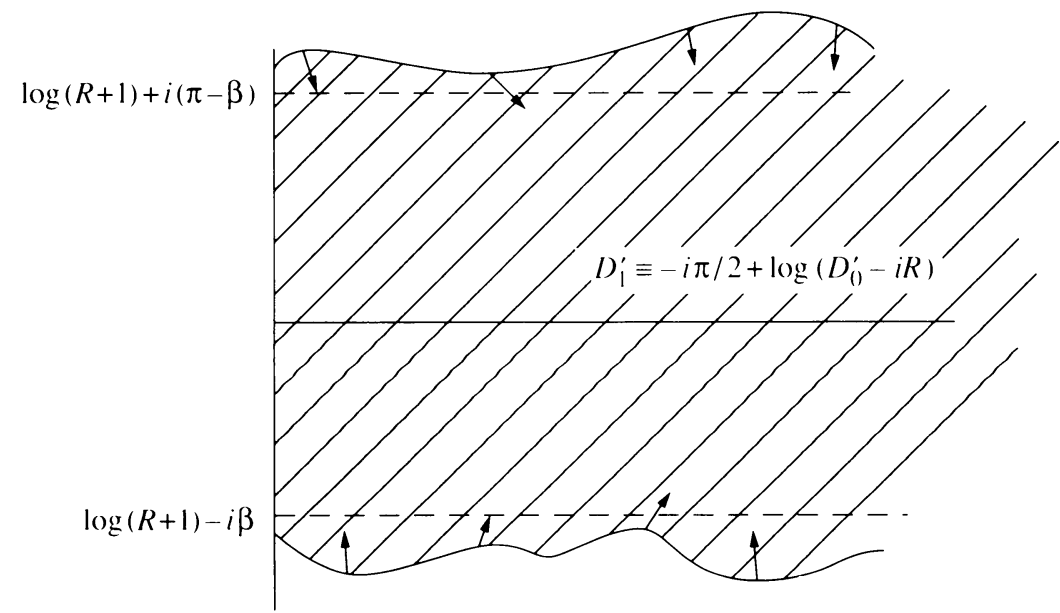

Figure 4 
motion with upward reflection in $\Psi(\mathbf{H})$ may be realised pathwise from a given Brownian motion $W$ in $\mathbf{C}$ by the recipe

$$
Z_{t}=z_{0}+W_{t}+i L_{t}
$$

where

$$
L_{1} \equiv \inf \left\{y \geq 0: \Psi(\mathbf{H})-i y \supseteq\left\{z_{0}+W_{u}: 0 \leq u \leq t\right\}\right\} .
$$

One may similarly realise the Brownian motion in the wedge with upward reflection on the boundaries as

$$
Z_{t}^{\prime}=z_{0}+W_{t}+i L_{t}^{\prime}
$$

where $L^{\prime}$ is defined as for $L$, with $C$ replacing $\Psi(\mathbf{H})$. Since $C \supseteq \Psi(\mathbf{H})$, one has always $L_{t}^{\prime} \leq L_{t}$. From the results of [VW] one know that the $\operatorname{Im}\left(Z_{t}^{\prime}\right) \rightarrow \infty$ a.s., and hence $\operatorname{Im}\left(Z_{t}\right) \rightarrow \infty$ a.s.

The argument for part (i) of the theorem is similar.

(ii) The problem of Brownian motion with variable directions of reflection in a cone in dimension $\geq 3$ has recently been tackled by Youngmee Kwon. I have not seen this interesting work yet, but am grateful to R. F. Bass for drawing my attention to its existence.

(iii) Does Brownian motion in the wedge actually reach the vertex in finite time? This interesting question will be treated in a forthcoming paper.

(iv) I am grateful to the referee for a number of very helpful remarks and suggestions.

(v) (Added in proof) Recently, K. Burdzy and D. Marshall (Hitting a boundary point with reflected Brownian motion, preprint) and the author (Brownian motion in a wedge with variable skew reflection. II, to appear in Proceedings of 1989 Evanston Conference on Stochastic Analysis, ed. M. Pinsky) have independently obtained by different methods necessary and sufficient conditions for the corner of the wedge to be approached. The author has also obtained necessary and sufficient integral criteria for the corner to be reached in finite time, and for extension of the process beyond the first hit on zero to be possible; these results will be the subject of a later paper.

\section{REFERENCES}

[DeB] R. D. De Blassie, Explicit semimartingale representation of Brownian motion in a wedge, preprint, 1988.

[D] E. B. Dynkin, Martin boundaries and non-negative solutions of a boundary value problem with a directional derivative, Uspekhi Mat. Nauk 19 (1964), 3-50.

[HLS] J. M. Harrison, H. J. Landau, and L. A. Shepp, The stationary distribution of reflected Brownian motion in a planar region, Ann. Probab. 13 (1985), 744-757.

[LeG] J.-F. LeGall, Mouvement brownien, cônes et processus stables, Probab. Theory Related Fields 76 (1987), 587-627.

[LS] P. L. Lions and A. S. Sznitman, Stochastic differential equations with reflecting boundary conditions, Comm. Pure Appl. Math. 37 (1984), 511-537.

[M] M. B. Malyutov, Brownian motion with reflection and the inclined derivative problem, Soviet Math. Dokl. 5 (1964), 822-825. 
[N] G. F. Newell, Approximate behaviour of tandem queues, Lecture Notes in Economics and Mathematical Systems, vol. 171, Springer, Berlin, 1979.

[ReW] M. I. Reiman and R. J. Williams, $A$ boundary property of semimartingale reflecting Brownian motion, Probab. Theory Related Fields 77 (1988), 87-97.

[RW] L. C. G. Rogers and D. Williams, Diffusions, Markov Processes and martingales, Vol. 2, Wiley, Chichester, 1987.

[R] L. C. G. Rogers, A guided tour through excursions, Bull. London Math. Soc. 21 (1989), 305-341.

[SV] A. S. Sznitman and S. R. S. Varadhan, A multidimensional process involving local time, Probab. Theory Related Fields 71 (1986), 553-579.

[TW] L. N. Trefethen and R. J. Williams, Conformal mapping solution of Laplace's equation on a polygon with oblique derivative boundary conditions, J. Comput. Appl. Math. 14 (1986), 227-249.

[VW] S. R. S. Varadhan and R. J. Williams, Brownian motion in a wedge with oblique reflection, Comm. Pure Appl. Math. 38 (1985), 405-443.

[W85a] R. J. Williams, Reflected Brownian motion in a wedge: semimartingale property, Probab. Theory Related Fields 69 (1985), 161-176.

[W85b] __, Recurrence classification and invariant measure for reflecting Brownian motion in a wedge, Ann. Probab. 13 (1985), 758-778.

[W87] __ Reflected Brownian motion with skew-symmetric data in a polyhedral domain, Probab. Theory Related Fields 75 (1987), 459-485.

Statistical laboratory, Department of Pure Mathematics and Mathematical Statistics, University of Cambridge, 16 Mill Lane, Cambridge CB2 1SB, UK

Current address: School of Mathematical Sciences, Queen Mary and Westfield College, University of London, Mile End Rd., London E1 4NS, U.K. 